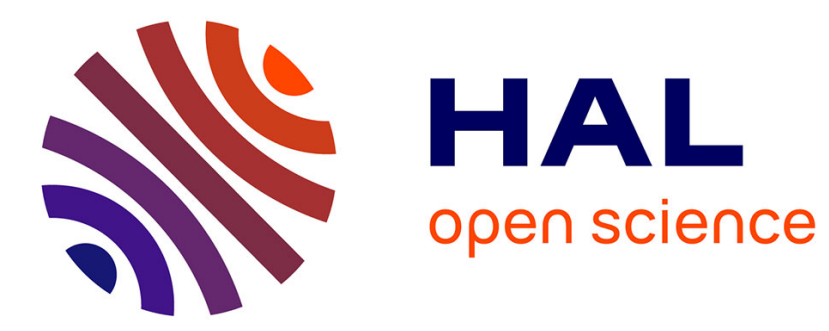

\title{
Première signalisation avérée du ravageur Melanagromyza obtusa (Malloch) dans la région néotropicale (Dipt., Agromyzidae)
}

\author{
Jean Etienne, Michel Martinez, Gladys Boëcasse
}

\section{- To cite this version:}

Jean Etienne, Michel Martinez, Gladys Boëcasse. Première signalisation avérée du ravageur Melanagromyza obtusa (Malloch) dans la région néotropicale (Dipt., Agromyzidae). Bulletin de la Société Entomologique de France, 2004, 109 (1), pp.105-106. hal-02677795

\section{HAL Id: hal-02677795 \\ https://hal.inrae.fr/hal-02677795}

Submitted on 31 May 2020

HAL is a multi-disciplinary open access archive for the deposit and dissemination of scientific research documents, whether they are published or not. The documents may come from teaching and research institutions in France or abroad, or from public or private research centers.
L'archive ouverte pluridisciplinaire HAL, est destinée au dépôt et à la diffusion de documents scientifiques de niveau recherche, publiés ou non, émanant des établissements d'enseignement et de recherche français ou étrangers, des laboratoires publics ou privés. 
NovoA F., 1975. - Los Carabidae de la Sierra de Guadarrama, I. Inventario de especies y biogeografía. Boletín de la Real Sociedad española de Historia Natural (Biología), 73: 99-147.

ORTiz A.S., Galián J., ANDÚJAR A. \& SERRAno J., 1989. - Estudio comparativo de la fauna de Carábidos de algunas lagunas de la región manchego-levantina. (España). (Coleoptera: Adephaga). Anales de Biología, Murcia, 15 (Biología Animal, 4-1988): 49-57.

ORTUÑo V. M. \& MARCOS J. M., 1997. - Carabidofauna (Insecta, Coleoptera) del área natural de Salburua (Vitoria, Álava). Ecología, 11: 457-482.

ORTUÑo V. M. \& TORIBIO M., 1996. - Los Coleópteros Carábidos, morfología, biología y sistemática. Fauna de la Comunidad de Madrid. Ministerio de Medio Ambiente, Organismo Autónomo de Parques Nacionales. 269 p.

- 2002. - Nuevos e interesantes datos para el catálogo de los Caraboidea (Coleoptera, Adephaga) de la Comunidad de Madrid. Boletín de la Sociedad entomológica Aragonesa, 31: 55-59.

REFSETH D., 1980. - Ecological analyses of carabid communities - potential use in biological classification for nature conservation. Biological Conservation, 17 (2): 131-141.

RIVAS-MARTíNEZ S., 1987. - Mapa de series de vegetación de España y memoria. Madrid. 29 mapas $(1: 400000)$ y 1 volumen $(268$ p.).

RuedA F. \& MONTES C., 1987. - Riparian carabids of saline aquatic ecosystems. Acta Phytophatologica et Entomologica Hungarica, 22 (1-4): 247-263.

SANTOS A., MONTES C. \& RAMíReZ-Díaz L., 1985. - Ciclos de vida de algunas poblaciones de carábidos (Col. Carabidae) de dos ecosistemas del bajo Guadalquivir (S.O. España) con especial referencia a Steropus globosus ebenus Quensel, 1806. Revue d'Ecologie et de Biologie du Sol, 22 (1): 75-95.

SAULEDA N., 1985. - Caraboidea amófilos y halófilos de la provincia de Alicante. Anales de la Universidad de Alicante. Escuela de Magisterio, 2: 241-264.

SERrano J., 1983. - Contribución al conocimiento de los Carábidos (Col.) de la Laguna de Gallocanta. Boletín de la Asociación española de Entomología, 6 (2): 369-379.

- 1989. - Adiciones al catálogo de los Carabidae (Coleoptera) de las Sierras de Guadarrama y Ayllón. Boletín de la Asociación española de Entomología, 13: 21-28.

SerRano J., Ortiz A. S. \& Galí́n J., 1990. - Los Carabidae de lagunas y ríos de la Submeseta Sur, España (Coleoptera, Adephaga). Boletín de la Asociación espáñola de Entomología, 14: 199-210.

VIVES J. \& VIVES E., 1978. - Coleópteros halófilos de Los Monegros. Boletín de la Asociación española de Entomología, 2: 205-214.

— 1981. - A propósit d'alguns coleóptes carábids de les zones salades espanyoles. II Sessió Conjunta d'Entomologia. Barcelona. p. 49-53.

- 1986. - IX. Carábidos (Ins. Coleoptera) de la laguna de Sariñena. 127-135. in C. Pedrocchi et al. : Estudio multidisciplinar de la Laguna de Sarieña (Huesca). Excma. Diputación de Huesca.

Zaballos J. P., 1986. - Notas fenológicas sobre los Carabidae (Coleoptera) de la península Ibérica. Anales de Biología, Murcia, 7 (Biología Animal, 2), 11-15.

Zaballos J. P. \& JEANNE C., 1994. - Nuevo catálogo de los carábidos (Coleoptera) de la península Ibérica. Monografías S.E.A.-1, Sociedad Entomológica Aragonesa., Zaragoza, 159 p.

Jean ÉTIENNE, Michel MARTINEZ \& Gladys BOËCASSE. - Première signalisation avérée du ravageur Melanagromyza obtusa (Malloch) dans la région néotropicale (Dipt., Agromyzidae)

Les Agromyzidae néotropicaux ont été récemment répertoriés (MARTINEZ \& ÉTIENNE, 2002) et ceux d'importance agronomique aux Antilles françaises ont fait également l'objet d'une liste commentée (ÉTIENNE et al., 1993). Il faut maintenant y ajouter l'espèce Melanagromyza obtusa (Malloch, 1914), présente aux Antilles. Cette mouche mineuse aurait cependant été interceptée à diverses occasions dans des bagages personnels de voyageurs (ANONYME [source Internet], 2001) aux USA et en République Dominicaine, en provenance de Puerto Rico, d'Haïti ou de République Dominicaine. Ainsi, bien qu'il paraisse évident, d'après cette donnée, que cette espèce se trouve effectivement dans ces trois pays et îles des Grandes Antilles, il n'existait jusqu'à présent aucune signalisation sur le terrain.

La première découverte in situ de Melanagromyza obtusa en région néotropicale a été faite à l'île de Marie-Galante (Grand-Bourg, Gagneron; G. Boëcasse leg., 15.IV.2002) où 10 a été 
obtenu d'une gousse de "pois d'Angole", comestible cultivé, Cajanus cajan (L.) Millsp. (Fabaceae). Les recherches de cette espèce se sont alors poursuivies et intensifiées dans cette île où des pupes extraites à nouveau de gousses de $C$. cajan de la même localité, le 8.II.2003, nous ont donné 4 9 .

Dès la découverte de cet Agromyzide à Marie-Galante, il a également été recherché en Guadeloupe. Après des contrôles infructueux pendant la période de pleine production (décembre 2002 et janvier 2003) il a été possible de le détecter pendant l'intercampagne (d'août à novembre 2003), période pendant laquelle les gousses de $C$. cajan restant sur pieds sont très peu nombreuses. Au total 67 individus de Melanagromyza obtusa ont été obtenus en Guadeloupe en 2003

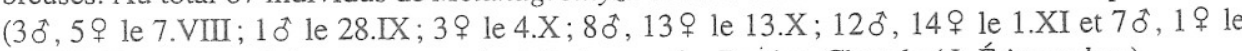
14.XI). Tous ces spécimens provenaient du Lamentin, Ravine-Chaude (J. Étienne leg.).

Cette mouche mineuse est présente dans la région orientale où elle est largement distribuée (Indes, Népal, Japon, Pakistan, Taiwan, Thailande, Vietnam, Philippines, Sri Lanka, Java,...) (SPENCER, 1973 ; SHANOWER et al., 1998) et dans la région australo-pacifique. Les citations de ce ravageur en Afrique sont erronées et se réferent en réalité à l'espèce Melanagromyza chalcosoma Spencer, 1959. Ces deux espèces sont proches par leur habitus mais leurs génitalia sont différents, bien que de même type ( $c f$. SPENCER, 1990, fig. 433 et 434, p. 124). Au regard de leur biologie les deux espèces sont également proches: elles se nymphosent dans les gousses de $C$. cajan après s'être développées aux dépens des graines; toutefois la larve de $M$. obtusa le fait hors de la graine tandis que celle de $M$. chalcosoma se pupifie à l'intérieur de celle-ci. Outre $C$. cajan ces deux espèces se nourrissent aussi d'autres Fabaceae : M. obtusa est signalé sur Flemingia $s p$. à Java et sur Phaseolus radiatus L. au Sri Lanka; $M$. chalcosoma est cité sur Flemingia rhodocarpa Bax et sur Vigna unguiculata (L.) Walp. en Afrique (SPENCER, 1973).

Bien que Melanagromyza obtusa soit apparemment bien présente à Marie-Galante et en Guadeloupe, ses dégâts, dans ces deux îles des Petites Antilles, paraissent pour le moment insignifiants, par rapport à ceux occasionnés par le lépidoptère Noctuidae Heliothis virescens (Fabricius), qui se développe aussi dans les gousses, au dépens des graines. Rappelons cependant que $M$. obtusa est un ravageur important de $C$. cajan dans la région orientale, en Indes notamment. Il convient donc de surveiller avec attention l'évolution de ses populations en Guadeloupe et à Marie-Galante, et de suivre sa dispersion dans la région néotropicale ; cela devrait inciter tous les pays de cette région à le rechercher. En effet Cajanus cajan est largement cultivé en région néotropicale ; aussi il est à craindre que la dispersion de ce nouvel arrivant soit rapide et invasive.

\section{AUTEURS CITÉS}

ANONYME, 2001. - Melanagromyza obtusa (Malloch). First report in the Western Hemisphere (Puerto Rico). Pest Alert, www.pestalert.org (5.X.2001): 2 p.

ÉTIENNE J., MARTINEZ M. \& HUC A., 1993. - Les mouches mineuses (Diptères, Agromyzides) d'importance agronomique aux Antilles. ANPP, Troisième Conférence Internationale sur les Ravageurs en Agriculture, Montpellier 7-8-9 décembre 1993, III : 1261-1272.

MARTINEZ M. \& ÉTIENNE J., 2002. - Liste systématique et biogéographique des Agromyzidae (Diptera) de la région néotropicale. Bollettino di Zoologia agraria e di Bachicoltura, Ser, II, 34 (1) : 25-52.

SHANOWER T.G., LAL S.S. \& BHAGWAT V.R., 1998. - Biology and management of Melanagromyza obtusa (Malloch) (Diptera: Agromyzidae). Crop Protection, 17 (3) : 249-263.

SPENCER K.A., 1973. - Agromyzidae (Diptera) of economic importance. Series Entomologica, 9, The Hague: W. Junk, 418 p.

1990. - Host Specialization in the world Agromyzidae (Diptera). Series Entomologica, 45, Kluwer Academic Publishers, Dordrecht, Netherlands : XI+444 p.

(J. É: INRA-URPV, Laboratoire d'Entomologie, domaine Duclos, F- 97170 Petit-Bourg, Guadeloupe, M.M. . INRA, UP, Écologie animale et Zoologie agricole, 2 place Pierre-Viala, F-34060 Montpellier cedex 01, G. B. : Laboratoire de la Protection des Végétaux, domaine Duclos, F-97170 Petit-Bourg, Guadeloupe). 